\title{
Các yếu tố tác động lên hành vi công dân tổ chức định hướng dịch vụ của nhân viên tại các doanh nghiệp ngành hàng không Việt Nam
}

\section{Factors influencing service - oriented organizational citizenship behavior of employees in Vietnam aviation enterprises}

\author{
Ngô Thị Thanh Huyền ${ }^{1 *}$ \\ ${ }^{1}$ Trường Học viện Hàng không Việt Nam, Việt Nam \\ "Tác giả liên hệ, Email: huyenngtt@vaa.edu.vn
}

THÔNG TIN

DOI: $10.46223 / \mathrm{HCMCOUJS.}$ econ.vi.14.1.504.2019

Ngày nhận: 25/10/2018

Ngày nhận lại: 30/11/2018

Duyệt đăng: 14/01/2019

\section{Tù khóa:}

hành vi công dân tổ chức định hướng dịch vụ, nhận thức được sự hỗ trợ của tổ chức, sự gắn kết công việc, sự hài lòng công việc, vốn tâm lý

\section{Keywords:}

job satisfaction, perceived organizational support, psychological capital,

\section{TÓM TÁT}

Khái niệm hành vi công dân tổ chức là một khái niệm mới trong các doanh nghiệp Việt Nam hiện nay đặc biệt là trong ngành dịch vụ khi mà vai trò của nhân viên được đặt lên hàng đầu. Xuất phát từ quan điểm trao đổi xã hội và dịch vụ nội bộ, nghiên cứu thảo luận về các yếu tố ảnh hưởng đến hành vi công dân tổ chức định hướng dịch vụ của nhân viên ngành hàng không. Nghiên cứu cung cấp lý thuyết, hướng dẫn thực tiễn cho ngành hàng không trong nước đang phát triển nhanh chóng cũng như những gợi ý cụ thể cho các doanh nghiệp trong ngành hàng không để phát triển hành vi tốt của nhân viên và để quản lý nhân viên một cách chiến lược. Kết quả phân tích hồi quy dựa trên mẫu khảo sát là 298 nhân viên làm việc trong các doanh nghiệp ngành hàng không Việt Nam cho thấy sự hài lòng công việc và sự gắn kết công việc có tác động mạnh mẽ đến những hành vi ngoài vai trò của nhân viên. Các yếu tố vốn tâm lý và nhận thức được sự hỗ trợ của tổ chức cũng ảnh hưởng không nhỏ đến những hành vi tích cực của nhân viên trong công việc. Kết quả này làm cơ sở cho các hàm ý quản trị tương ứng.

\section{ABSTRACT}

Today the concept of organizational citizenship behavior is a new concept in Vietnamese enterprises especially in the service sector whereas employees is are always at the forefront of enterprises. Based on the point of view of social exchange and internal services, this study identifies factors that affect serviceoriented organizational citizenship behavior of aviation personnel. This study provides theoretical and practical implications for the aviation industry in developing countries as well as specific suggestions for aviation enterprises to develop organizational behavior of employees and to manage them strategically. The sample includes 298 employees working in the Vietnamese aviation industry. The regression analysis is employed to explore job 
service - oriented organizational citizenship behavior, work engagement satisfaction and work engagement that have a strong influence on extra-role behaviors. The psychological capital factors and perceived organizational support also significantly affect the positive behavior of employees at work. Discussions and implications are presented along these statistical results.

\section{Giới thiệu}

Trong sự phát triển của ngành dịch vụ trên toàn thế giới, các doanh nghiệp không chỉ phải đối mặt với những vấn đề sử dụng nguồn tài nguyên môi trường và phát triển sản phẩm mới mà còn phải quan tâm đến các nguồn lực gắn với vốn con người, công nghệ hoặc tài sản hữu hình; Hơn nữa, làm cho chúng trở thành nguồn tài nguyên có giá trị trong sự đổi mới và cạnh tranh. Trong ngành công nghiệp dịch vụ, đội ngũ nhân viên chính là một trong số các nguồn lực đó, họ trực tiếp tiếp xúc với khách hàng có thể được coi là đại diện của công ty. Ngoài việc cung cấp cho khách hàng các dịch vụ vượt trội, họ phải thực hiện các nhiệm vụ trong vai trò của mình trong tổ chức và phải sẵn sàng nỗ lực để tăng cường hoạt động và duy trì hình ảnh tổ chức (Podsakoff \& MacKenzie, 1997). Bên cạnh đó, đối mặt với cạnh tranh trong ngành dịch vụ hiện nay sẽ rất khó khăn để đáp ứng nhu cầu đa dạng và thay đổi nhanh chóng của khách hàng nếu nhân viên phục vụ không cung cấp một dịch vụ tốt và có chất lượng. Để cung cấp dịch vụ có chất lượng và làm hài lòng khách hàng đòi hỏi nhân viên phải vượt ra khỏi bản mô tả công việc, tận tâm và có nhiều ý tưởng sáng tạo trong công việc (Ma \& Qu, 2011).

Từ các nghiên cứu trước đây cho thấy tầm quan trọng của các hành vi ngoài vai trò của các nhân viên được gọi là các hành vi công dân tổ chức (Bateman \& Organ, 1983). Các nghiên cứu gần đây của hành vi công dân tổ chức đã dần được chuyển sang ngành dịch vụ và nhân viên. Các hành vi công dân tổ chức định hướng dịch vụ có một tầm quan trọng rất lớn trong doanh nghiệp và được thể hiện qua lòng trung thành, sự tham gia vào công việc và quá trình cung cấp dịch vụ (Bettencourt, Gwinner, \& Meuter, 2001; Podsakoff \& MacKenzie, 1997). Hành vi công dân tổ chức theo định hướng dịch vụ trong ngành dịch vụ không chỉ mang lại dịch vụ tốt, cung cấp dịch vụ chất lượng cao hơn, tạo môi trường thân thiện khuyến khích sự tương tác của khách hàng mà còn cung cấp một cách nhìn đổi mới dịch vụ nhằm đáp ứng nhu cầu khách hàng tốt hơn và tạo sự hài lòng cao hơn (Podsakoff, MacKenzie, Paine, \& Bacharch, 2009).

Mặc dù hành vi công dân tổ chức ngày càng được áp dụng nhiều cho ngành dịch vụ, nhưng có rất ít nghiên cứu ứng dụng trong trường hợp nhân viên của ngành hàng không. Khác với các nghiên cứu khác về trao đổi xã hội, nghiên cứu này tập trung vào thực tế ngành hàng không dựa vào sự hợp tác và hỗ trợ giữa các đồng nghiệp để cung cấp dịch vụ có chất lượng cao.

\section{Cơ sở lý thuyết và mô hình nghiên cứu}

\section{Hành vi công dân tổ chức định hướng dịch vụ}

Bateman và Organ (1983) đã sử dụng thuật ngữ hành vi công dân tổ chức cho những nghiên cứu đầu tiên của mình. Họ định nghĩa khái niệm này như một phần các biện pháp cho người lao động nhằm nâng cao năng suất, tinh thần đoàn kết và sự gắn kết ở nơi làm việc. 
Nghiên cứu ban đầu của lĩnh vực này, chủ yếu là xác định trách nhiệm và hành vi của nhân viên, mà hầu như là không được đánh giá trong hiệu quả công việc, nhưng chúng có đóng góp trong việc cải thiện hiệu quả của tổ chức (Bienstock, Demoranville, \& Smith, 2003, p. 360). Năm 1988, Organ đã làm rõ bản chất của hành vi công dân tổ chức hơn thông qua định nghĩa sau: "Hành vi công dân tổ chức là những hành vi mang tính cá nhân, tự nguyện vượt lên khỏi vai trò nhiệm vụ được mô tả trong công việc, với mục đích không phải để kiếm phần thưởng của tổ chức mà góp phần nâng cao hiệu quả công việc của tổ chức và không được thể hiện rõ ràng trong quy định của tổ chức" (Organ, 1988, p. 4).

Các tổ chức dịch vụ thường bị thách thức bởi nhu cầu độc đáo và nhất thời của khách hàng, cũng như phải đối phó với nhiều đối tượng khách hàng khác nhau (Prentice \& King, 2011). Nên nhờ môi trường riêng biệt của các tổ chức dịch vụ, Bettencourt và cộng sự (2001) đã thực hiện một nghiên cứu trong ngành hàng không tập trung vào đối tượng nhân viên những người trực tiếp cung cấp dịch vụ và đưa ra được khái niệm hành vi công dân tổ chức trong ngành hàng không. Gần đây nhất là nghiên cứu của Tsai và Su (2011, pp. 1916-1917) chỉ ra rằng: "Hành vi công dân tổ chức định hướng dịch vụ là những hành vi định hướng vào việc cung cấp một dịch vụ có chất lượng. Theo đó, những nhân viên được coi là những người đại diện cho tổ chức với bên ngoài, họ có thể làm tăng hoặc giảm hình ảnh của tổ chức". Do vậy, điều quan trọng của tổ chức là khuyến khích các nhân viên tham gia vào công việc và phát huy lòng trung thành của mình không chỉ đối với các sản phẩm và dịch vụ mà còn hình ảnh của tổ chức.

Theo Blau (1964), lý thuyết trao đổi xã hội là nền tảng khung lý thuyết và nhấn mạnh vai trò quan trọng trong sự tương tác giữa vốn tâm lý của nhân viên, sự gắn kết của nhân viên, sự hài lòng và sự hỗ trợ của tổ chức đối với nhân viên để nhân viên có được hành vi tích cực trong tổ chức. Theo Saks (2006) một lý do mạnh mẽ có thể giải thích tại sao các cá nhân có mức độ gắn kết khác nhau và có sự khác biệt về kết quả làm việc của từng cá nhân đó chính là sự gắn kết liên quan đến mối quan hệ hai chiều giữa người sử dụng lao động và người lao động. Lý thuyết này đã cung cấp một cơ sở để hiểu rõ vai trò và mối quan hệ giữa nhân viên và nhà quản trị với tổ chức. Các mối quan hệ trao đổi xã hội có vai trò làm tăng mức độ cam kết của nhân viên đối với tổ chức và nghĩa vụ của tổ chức đối với nhân viên.

\section{Vốn tâm lý và hành vi công dân tổ chức định hướng dịch vụ}

Có nhiều cách hiểu về vốn tâm lý. Theo Avey, Luthans, và Jensen (2009) chỉ ra rằng Vốn tâm lý được định nghĩa như là bản chất con người và là trạng thái tâm lý tích cực trong sự phát triển của cá nhân. Vốn tâm lý trả lời cho câu hỏi bạn là ai và bạn có thể đạt được cái gì xét về mặt phát triển tích cực. Vốn tâm lý ảnh hưởng tích cực tới bản chất con người và giúp cá nhân có được hiệu quả cao trong công việc (Lusthans \& Youssef, 2004). Nói chung vốn tâm lý thường được nghiên cứu dưới cái ô lý thuyết là hành vi tổ chức.

Theo Luthans, Avolio, Walumbwa, và Li (2005) có một mối quan hệ tích cực giữa vốn tâm lý đến kết quả thực hiện của tổ chức. Trong nghiên cứu Avey và cộng sự (2009) cho thấy có một mối quan hệ tích cực giữa vốn tâm lý và hành vi công dân tổ chức, theo đó các cá nhân có vốn tâm lý tích cực sẽ có khuynh hướng tự tin hơn và có những hành vi tích cực đối với tổ chức. Điều này cũng được nhấn mạnh trong nghiên cứu của Walumbwa và Schaubroeck (2009) 
nghiên cứu về lãnh đạo đích thực, vốn tâm lý và hành vi công dân tổ chức. Các nghiên cứu tiếp theo của Khosravizadeh và cộng sự (2017) và Manish và Prathap (2017) tập trung vào việc xác định tổ chức như một người điều tiết mối quan hệ giữa vốn tâm lý trong mối quan hệ với nhân viên và hành vi công dân tổ chức. Rõ ràng là các nhân viên có vốn tâm lý tích cực và có sự nhận diện với tổ chức một cách mạnh mẽ thì sẽ có những hành vi công dân tổ chức tốt hơn. Ta có giả thuyết sau:

\section{H1: Vốn tâm lý có tác động tích cực lên hành vi công dân tổ chức định hướng dịch vu}

\section{Sụ gắn kết công việc và hành vi công dân tổ chức định hướng dịch vụ}

Trong hai thập kỷ qua, đã có một sự quan tâm đáng kể về sự gắn kết công việc của các nhà nghiên cứu trên toàn cầu (Albrecht, 2010; Saks, 2006). Sự gắn kết công việc đang nổi lên như là một trong những yếu tố chính để nâng cao hiệu quả hoạt động của nhân viên và tăng trưởng tổ chức. Nhân viên khi có sự gắn kết với công việc họ có ít hành vi tiêu cực hơn trong tổ chức (e.g., nghỉ việc hay dự định nghỉ việc). Mặt khác, họ có liên quan về thể chất và cảm xúc với công việc của họ, có động lực mạnh mẽ và sẵn sàng cải thiện kỹ năng và kiến thức liên quan đến công việc (Bakker, 2011; Schaufeli, 2012). Schaufeli, Salanova, Gonzalez-Roma, và Bakker (2002, p. 74) đã xác định sự gắn kết công việc là "Một trạng thái tinh thần tích cực liên quan đến hoàn thành công việc của nhân viên được đặc trưng bởi sự hăng hái, sự cống hiến, sự say mê".

Nghiên cứu của Kang (2014) kiểm tra mối quan hệ giữa các yếu tố vốn tâm lý, môi trường dịch vụ, sự gắn kết công việc, sự hài lòng của nhân viên, hành vi công dân tổ chức và ý định nghỉ việc. Kết quả nghiên cứu cho thấy vốn tâm lý có tác động tích cực đến sự gắn kết công việc và từ đó có ảnh hưởng đến hành vi công dân tổ chức. Ta có giả thuyết:

H2: Sụ gắn kết công việc có tác động tích cực lên hành vi công dân tổ chức định hướng dịch $v$ u

\section{Sụ hài lòng công việc và hành vi công dân tổ chức định hướng dịch vụ}

Khái niệm về sự hài lòng công việc lần đầu tiên được phát triển từ những nghiên cứu của Hawthorne vào cuối những năm 1920 và đầu những năm 1930 của Elton Mayo tại nhà máy Hawthorne của Công ty Western Electric ở Chicago. Kết quả là cảm xúc của nhân viên có thể ảnh hưởng đến hành vi làm việc của họ. Các mối quan hệ xã hội và các yếu tố tâm lý là những nguyên nhân chính dẫn đến sự hài lòng và năng suất trong nhân viên. Cranny, Smith, và Stone (1992) đã đưa ra định nghĩa tổng quát chung về sự hài lòng công việc, đó là sự kết hợp của những hành động phản ứng lại thuộc về tình cảm nhận thức của người lao động đối với công việc dựa trên việc so sánh kết quả công việc thực tế với kết quả công việc được mong đợi.

Nghiên cứu của Tsai và Su (2011) về "Sự ảnh hưởng của phong cách lãnh đạo, sự hài lòng công việc đến hành vi công dân tổ chức theo định hướng dịch vụ của tiếp viên hàng không". Nghiên cứu này đã điều tra mối quan hệ giữa lãnh đạo, sự hài lòng công việc và các hành vi công dân tổ chức định hướng dịch vụ cho các tiếp viên hàng không. Kết quả cho thấy sự lãnh đạo và sự hài lòng trong công việc có liên quan một cách tích cực đến hành vi công dân tổ chức định hướng dịch vụ. Mối quan hệ này cũng được chỉ ra trong nghiên cứu của Ikonne (2015) khi 
ông nghiên cứu về hành vi của các nhân viên thư viện tại trường đại học Negiria. Ta có giả thuyết:

H3: Sự hài lòng công việc có tác động tích cực lên hành vi công dân tổ chức định hướng dịch $v$ u

\section{Nhận thức đự̣c sụ hỗ trọ̣ của tổ chức và hành vi công dân tổ chức định hướng dịch vụ}

Nhận thức được sự hỗ trợ của tổ chức dựa trên quan điểm của lý thuyết trao đổi xã hội và đã thu hút được sự quan tâm nghiên cứu đáng kể trong lĩnh vực hành vi tổ chức. Theo Eisenberger, Huntington, Hutchison, và Sowa (1986) và Rhoades và Eisenberger (2002) chỉ ra rằng Nhận thức được sự hỗ trợ tổ chức là mức độ mà nhân viên tin rằng tổ chức của họ đánh giá cao đóng góp của họ và quan tâm đến đời sống tinh thần của họ.

Nhận thức được sự hỗ trợ của tổ chức đã được chấp nhận như là một yếu tố quan trọng ảnh hưởng đến thái độ và hành vi công việc của cá nhân. Các nghiên cứu trước đây cho thấy rằng nhận thức được sự hỗ trợ của tổ chức tích cực ảnh hưởng đến sự hài lòng công việc (Eisenberger, Cummings, Armeli, \& Lynch, 1997), cam kết tổ chức (Eisenberger, Fasolo, \& Davis-LaMastro, 1990), và hành vi trong vai trò và hành vi ngoài vai trò (Rhoades \& Eisenberger, 2002). Ngoài ra, một số nghiên cứu cho thấy rằng nhận thức được sự hỗ trợ của tổ chức có liên quan tiêu cực đến các hành vi tiêu cực của nhân viên như vắng mặt, trễ hẹn... (Rhoades \& Eisenberger, 2002). Tóm lại, mức nhận thức được sự hỗ trợ của tổ chức cao giúp ích cho việc tăng kết quả làm việc tích cực và giảm các kết quả tiêu cực (Ali, 2014). Ta có giả thuyết:

H4: Nhận thức được sụ hỗ trợ của tổ chức có tác động tích cực lên hành vi công dân tổ chức định huớng dịch vu

Từ các phân tích ở trên, mô hình nghiên cứu được đề xuất như sau: 


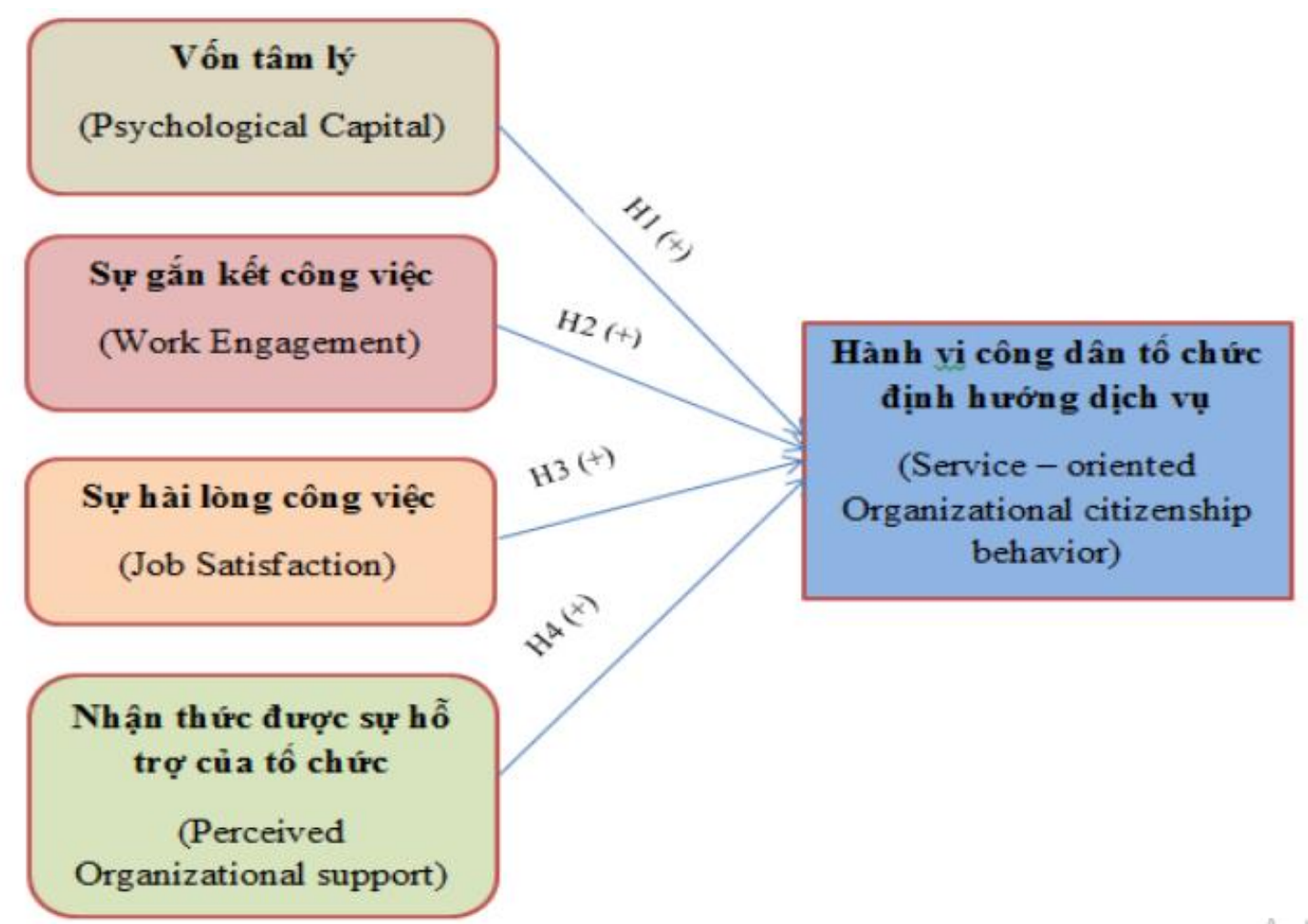

Hình 1. Mô hình nghiên cứu

\section{Phương pháp nghiên cứu}

Nghiên cứu được thực hiện qua hai giai đoạn, nghiên cứu sơ bộ và nghiên cứu chính thức. Nghiên cứu sơ bộ định lượng với 151 nhân viên được thực hiện vào tháng 10/2017. Từ kết quả nghiên cứu này, một số phát biểu trong thang đo đã được hiểu chỉnh/bổ sung phù hợp với bối cảnh nghiên cứu các doanh nghiệp ngành hàng không Việt Nam. Cụ thể thang đo Vốn tâm lý của Luthans, Avolio, Avey, và Norman (2007) gồm 9 biến quan sát; Thang đo Sự gắn kết công việc của Schaufeli và cộng sự (2002) gồm 8 biến quan sát; Thang đo Sự hài lòng công việc của Schriesheim và Tsui (1980) gồm 6 biến quan sát; Thang đo Nhận thức được sự hỗ trợ của tổ chức của Rhoades và Eisenberger (2002) gồm 6 biến quan sát và Thang đo hành vi công dân tổ chức định hướng dịch vụ của Bettencourt, Gwinner, và Meuter (2001) gồm 9 biến quan sát.

Nghiên cứu chính thức được thực hiện trong 2 tuần cuối tháng 12/2017 với 298 nhân viên. Nghiên cứu sử dụng phương pháp lấy mẫu thuận tiện, phi xác xuất để tiếp cận nhân viên và phỏng vấn trực tiếp bằng phiếu khảo sát có cấu trúc. Thang đo sử dụng là thang Likert 5 điểm. Phương pháp phân tích dữ liệu sử dụng phần mềm SPSS 20.0.

\section{Kết quả nghiên cứu}

\section{Mô tả thông tin mẫu}

Trong bộ dữ liệu thu thập từ 298 nhân viên có có 90 nam (chiến 30,2\%) và 208 nữ (chiếm 69,8\%). Các đặc trưng khác của mẫu dữ liệu được trình bày trong Bảng 1 . 


\section{Bảng 1}

Mô tả mẫu khảo sát

\begin{tabular}{|c|c|c|c|c|c|}
\hline Trình độ học vấn & 298 & $\%$ & Độ tuổi & 298 & $\%$ \\
\hline Phổ thông & 11 & 3,69 & Dưới 25 & 72 & 24,16 \\
\hline Trung cấp & 22 & 7,38 & Từ 25 đến 35 & 90 & 30,20 \\
\hline Cao đẳng & 135 & 45,30 & Từ 35 đến 45 & 85 & 28,52 \\
\hline Đại học & 118 & 39,60 & Trên 45 & 51 & 17,12 \\
\hline Trên đại học & 12 & 4,03 & \multirow{2}{*}{ Kinh nghiệm làm việc } & \multirow{2}{*}{298} & \multirow{2}{*}{$\%$} \\
\hline Thu nhập & 298 & $\%$ & & & \\
\hline Dưới 5 triệu & 31 & 10,04 & Dưới 3 năm & 180 & 60,40 \\
\hline Từ 5 đến 10 triệu & 43 & 14,43 & Từ 3 đến 10 năm & 70 & 23,50 \\
\hline Từ 11 đến 15 triêu & 104 & 34,90 & \multirow{2}{*}{ Trên 10 năm } & \multirow{2}{*}{48} & \multirow{2}{*}{16,10} \\
\hline Trên 15 triệu & 120 & 40,27 & & & \\
\hline
\end{tabular}

Nguồn: Kết quả phân tích dữ liệu của nhóm nghiên cứu

Phần kiểm định thang đo được thực hiện qua phân tích độ tin cậy Cronbach's Alpha cho thấy các biến PC8, PC9, WE8, SOCB1 có hệ số tương quan biến tổng nhỏ hơn mức cho phép (0.3). Kết quả kiểm định Cronbach’s Alpha được trình bày trong Bảng 2 .

\section{Bảng 2}

Kết quả kiểm định Cronbach’s Alpha

\begin{tabular}{|c|c|}
\hline Khái niệm và các biến & $\begin{array}{c}\text { Cronbach's } \\
\text { Alpha nếu loại } \\
\text { biến }\end{array}$ \\
\hline \multicolumn{2}{|l|}{ 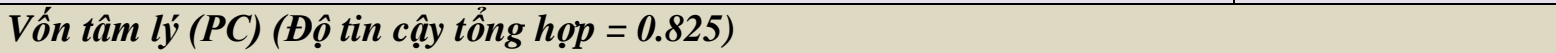 } \\
\hline PC1 Tôi cảm thấy tự tin khi giải quyết vấn đề rắc rối trong công việc. & .785 \\
\hline PC2 Tôi cảm thấy tự tin khi giao tiếp trong công việc. & .795 \\
\hline PC3 Khi gặp khó khăn, tôi luôn suy nghĩ theo hướng tích cực & .794 \\
\hline PC4 Nhìn chung, Tôi luôn mong đợi mọi thứ tốt đẹp sẽ đến với tôi & .786 \\
\hline PC5 Tôi luôn nỗ lực theo đuổi để đạt được mục tiêu & .805 \\
\hline $\begin{array}{l}\text { PC6 Tôi nhanh chóng lấy lại tinh thần để vượt qua những khó khăn trong công } \\
\text { việc }\end{array}$ & .810 \\
\hline PC7 Tôi luôn nhiệt tình giúp đỡ đồng nghiệp trong công việc & .794 \\
\hline PC8 Tôi vượt qua sự tức giận một cách nhanh chóng & .844 \\
\hline PC9 Tôi luôn cố gắng làm việc dưới áp lực cao của công việc & .847 \\
\hline \multicolumn{2}{|l|}{ Sụ hài lòng công việc (JS) (Độ tin cậy tổng hợp = 0.813) } \\
\hline JS1 Tôi hài lòng với tính chất công việc tôi đang làm & .805 \\
\hline JS2 Tôi hài lòng với người quản lý của tôi & .773 \\
\hline JS3 Tôi hài lòng về các mối quan hệ với các đồng nghiệp & .792 \\
\hline
\end{tabular}




\begin{tabular}{|c|c|}
\hline Khái niệm và các biến & $\begin{array}{c}\text { Cronbach's } \\
\text { Alpha nếu loại } \\
\text { biến } \\
\end{array}$ \\
\hline JS4 Tôi hài lòng về mức tiền lương được nhận được từ công việc & .787 \\
\hline JS5 Tôi hài lòng với các cơ hội nhận được, sự thăng tiến và phần thưởng & .790 \\
\hline JS6 Xét về mọi mặt, tôi hài lòng về tình trạng công việc hiện tại & .755 \\
\hline \multicolumn{2}{|l|}{ Sụ gắn bó với công việc (WE) (Độ tin chậy tổng hợp = 0.842) } \\
\hline WE1 Tôi cảm thấy tràn đầy năng lượng khi làm việc & .787 \\
\hline WE2 Mỗi sáng khi thức dậy, tôi mong muốn đến nơi làm việc & .785 \\
\hline WE3 Tôi có thể làm việc liên tục mà không nghỉ ngơi khi cần thiết & .783 \\
\hline WE4 Tôi luôn hoàn thành tốt công việc ngay cả khi mọi việc không suôn sẻ & .773 \\
\hline WE5 Tôi tự hào về những việc tôi làm & .814 \\
\hline WE6 Tôi nhiệt tình với công việc của tôi & .776 \\
\hline WE7 Công việc truyền cảm hứng cho tôi & .814 \\
\hline WE8 Tôi sẵn sàng làm những công việc mang tính thử thách & .842 \\
\hline \multicolumn{2}{|l|}{ Nhận thức đự̂c sụ hỗ trọ của tổ chức (OP) (Độ tin cậy tổng hợp=0.847) } \\
\hline OP1 Tổ chức rất quan tâm đến việc tôi hoàn thành các công việc được giao & .832 \\
\hline OP2 Tổ chức rất quan tâm đến tôi & .809 \\
\hline OP3 Tổ chức coi trọng những đóng góp của tôi đối với tổ chức & .835 \\
\hline OP4 Tổ chức rất lưu tâm đến các mục tiêu và quan điểm của tôi & .831 \\
\hline OP5 Tổ chức sẵn sàng giúp đỡ tôi nếu tôi cần được ưu đãi đặc biệt & .800 \\
\hline OP6 Tổ chức tạo điều kiện cho tôi học tập để nâng cao trình độ & .819 \\
\hline \multicolumn{2}{|l|}{ Hành vi công dân tổ chức định hướng dịch vụ (SOCB) (Độ tin cậy tổng hợp=0.839) } \\
\hline SOCB1 Tôi nói tốt về tổ chức với những người khác & .839 \\
\hline SOCB2 Tôi luôn chủ động cải tiến dịch vụ của tổ chức & .779 \\
\hline SOCB3 Tôi khuyến khích bạn bè, gia đình sử dụng dịch vụ của tổ chức & .781 \\
\hline SOCB4 Tôi tận tâm khi phục vụ khách hàng & .821 \\
\hline SOCB5 Tôi kịp thời đáp ứng các yêu cầu của khách hàng & .817 \\
\hline SOCB6 Tôi luôn có thái độ tích cực trong công việc & .798 \\
\hline SOCB7 Tôi cố gắng làm việc một cách tỉ mỉ và cẩn trọng & .798 \\
\hline SOCB8 Tôi luôn có nhiều ý tưởng sáng tạo trong công việc & .802 \\
\hline SOCB9 Tôi khuyến khích đồng nghiệp xây dựng ý tưởng trong việc cải tiến dịch vụ & .811 \\
\hline
\end{tabular}

Nguồn: Kết quả xử lý từ dữ liệu điều tra

Kết quả phân tích nhân tố khám phá EFA cho các biến độc lập cho thấy các biến quan sát có tương quan với nhau trong tổng thể $(\mathrm{Sig}=0.000<0.5)$. Hệ số $\mathrm{KMO}=0.902>0.5$ : Phân tích nhân tố thích hợp với dữ liệu nghiên cứu. Có 4 nhân tố được trích ra từ phân tích EFA. Giá trị Eigenvalues = $1.737>1$ là đạt yêu cầu. Giá trị tổng phương sai trích: 57.209 (đạt yêu cầu). Hầu hết các biến quan sát đều có hệ số tải nhân tố $>0.5$. Tuy nhiên có 2 biến quan sát là WE5 và WE7 có hệ số tải nhân tố thấp hơn 0.5 . Do mẫu khảo sát khá lớn (298 mẫu) nên hệ số tải nhân tố lớn hơn 0.4 là có thể chấp nhận (Nguyễn Định Thọ, 2013). Bên cạnh đó, xét theo ý nghĩa quản trị và trong ngành hàng không thì tác giả giữ lại 2 biến quan sát này là: "Tôi tự hào về những việc tôi làm" và "Công việc truyền cảm hứng cho tôi”. Theo nghiên cứu định tính của 
tác giả khi phỏng vấn nhóm thì cả nhóm nam và nữ đều nhận định rằng bản thân nhân viên tự hào với công việc cũng như công việc truyền được cảm hứng cho họ đóng vai trò quan trọng để họ có được những hành vi tốt trong công việc. Nên tác giả sẽ giữ lại 2 biến quan sát này vì lý do nêu trên.

Kết quả phân tích nhân tố khám phá EFA cho biến phụ thuộc cho thấy các biến quan sát có tương quan với nhau trong tổng thể $(\mathrm{Sig}=0.000<0.5)$. Hệ số $\mathrm{KMO}=0.730>0.5$ : Phân tích nhân tố thích hợp với dữ liệu nghiên cứu. Có 1 nhân tố được trích ra từ phân tích EFA. Giá trị Eigenvalues $=4.038>1$ : đạt yêu cầu. Giá trị tổng phương sai trích: 50.479 (đạt yêu cầu). Tất cả các biến quan sát đều có hệ số tải nhân tố > 0.5: đạt yêu cầu. Như vậy, thang đo "Hành vi công dân tổ chức định hướng dịch vụ" đạt giá trị hội tụ.

Kết quả phân tích nhân tố cho thấy các biến độc lập và biến phụ thuộc trong mô hình đều đạt giá trị hội tụ và giá trị phân biệt chấp nhận được. Phân tích EFA là thích hợp với dữ liệu nghiên cứu. Có 5 nhân tố được trích ra từ kết quả phân tích bao gồm 34 biến quan sát.

\section{Phân tích hồi quy}

Kết quả phân tích hệ số tương quan cho thấy tương quan giữa biến phụ thuộc với các biến độc lập khá cao. Lần lượt là Vốn tâm lý (PC) là 0.480 ; Sự hài lòng công việc (JS) là 0.536 ; Sự gắn kết công việc (WE) là 0.561 ; Nhận thức được sự hỗ trợ của tổ chức $(\mathrm{OP})$ là 0.507 . Như vậy biến phụ thuộc có mối quan hệ tuyến tính với các biến độc lập. Vì vậy, các biến độc lập này có thể đưa vào mô hình để giải thích cho biến hành vi công dân tổ chức định hướng dịch vụ.

\section{Bảng 3}

Tóm tắt mô hình hồi quy

\begin{tabular}{|c|c|c|c|c|}
\hline Mô hình & $\mathbf{R}$ & $\mathbf{R}$ bình phương & $\begin{array}{c}\text { R bình phương } \\
\text { điều chỉnh }\end{array}$ & $\begin{array}{c}\text { Sai số chuẩn của } \\
\text { dự báo }\end{array}$ \\
\hline 1 & $.724^{\mathrm{a}}$ & .524 & .517 & .34445 \\
\hline
\end{tabular}

Nguồn: Kết quả xử lý từ dữ liệu điều tra

\section{Bảng 4}

Hệ số hồi quy mô hình

\begin{tabular}{|c|c|c|c|c|c|c|c|}
\hline \multirow{2}{*}{ Mô hình } & \multicolumn{2}{|c|}{ Hệ số chưa chuẩn hóa } & \multirow{2}{*}{$\begin{array}{c}\begin{array}{c}\text { Hệ số } \\
\text { chuẩn hóa }\end{array} \\
\text { Beta }\end{array}$} & \multirow{2}{*}{$\mathbf{t}$} & \multirow{2}{*}{ Sig. } & \multicolumn{2}{|c|}{$\begin{array}{c}\text { Thống kê đa cộng } \\
\text { tuyến }\end{array}$} \\
\hline & B & $\begin{array}{l}\text { Sai số } \\
\text { chuẩn }\end{array}$ & & & & Dung sai & VIF \\
\hline (Hằng số) & .664 & .155 & & 4.296 & .000 & & \\
\hline $\mathrm{PC}$ & .107 & .042 & .130 & 2.533 & .012 & .618 & 1.619 \\
\hline JS & .305 & .037 & .356 & 8.277 & .000 & .878 & 1.139 \\
\hline WE & .211 & .039 & .277 & 5.411 & .000 & .622 & 1.607 \\
\hline OP & .168 & .035 & .228 & 4.798 & .000 & .720 & 1.389 \\
\hline
\end{tabular}

Nguồn: Kết quả xử lý từ dữ liệu điều tra 
Kiểm định $\mathrm{F}$ về tính phù hợp của mô hình hồi quy tuyến tính tổng thể. Điều này cho chúng ta biết biến phụ thuộc có tương quan tuyến tính với toàn bộ biến độc lập hay không. Đặt giả thuyết $\mathrm{H} 0$ là $\beta 0=\beta 1=\beta 2=\beta 3=\beta 4$. Trong bảng thống kê Anova ta có giá trị sig $=0.000$ $(<5 \%)$, nên cho phép ta có thể bác bỏ giả thuyết $\mathrm{H} 0$. Điều này có ý nghĩa là các biến độc lập trong mô hình có tương quan tuyến tính với biến phụ thuộc tức là kết hợp của các biến độc lập có thể giải thích được sự thay đổi của biến phụ thuộc.

Trong bảng tóm tắt mô hình ta thấy hệ số $\mathrm{R}^{2}$ đã hiệu chỉnh bằng 0.517 (51.7\%) nghĩa là mô hình hồi quy tuyến tính đã xây dựng phù hợp với dữ liệu $51.7 \%$, hay nói cách khác hơn là bốn biến độc lập giải thích được $51.7 \%$ phương sai của biến phụ thuộc là hành vi công dân tổ chức định hướng dịch vụ.

Phân tích hồi quy cho ta phương trình hồi quy tuyến tính đã chuẩn hóa như sau:

$$
\mathrm{SOCB}=0.305 * \mathrm{JS}+0.211 * \mathrm{WE}+0.168 * \mathrm{OP}+0.107 * \mathrm{PC}+0.664
$$

\section{Trong đó}

SOCB: Hành vi công dân tổ chức định hướng dịch vụ

PC: Vốn tâm lý

JS: Sự hài lòng công việc

WE: Sự gắn bó công việc

OP: Nhận thức được sự hỗ trợ của tổ chức

\section{Thảo luận kết quả nghiên cứu}

Các kết quả phân tích thống kê ở trên làm cơ sở cho một số nhận định sau:

Vốn tâm lý có tác động tích cực đến hành vi công dân tổ chức định hướng dịch vụ, từ kết quả hồi quy ta thấy trọng số hồi quy Beta $=0.130$ và mang dấu dương, $\mathrm{p}=0.012<0.05$. Điều này có nghĩa là một nhân viên có vốn tâm lý tích cực thì họ sẽ có những hành vi công dân tổ chức định hướng dịch vụ tốt và ngược lại.

Sự gắn kết công việc có tác động tích cực đến hành vi công dân tổ chức định hướng dịch vụ, từ kết quả hồi quy ta thấy trọng số hồi quy Beta $=0.277, \mathrm{p}=0.00<0.05$. Điều này có nghĩa là một nhân viên có Sự gắn kết với công việc cao thì sẽ có những hành vi công dân tổ chức định hướng dịch vụ tốt và ngược lại.

Sự hài lòng công việc có tác động tích cực đến hành vi công dân tổ chức định hướng dịch vụ, từ kết quả hồi quy ta thấy trọng số hồi quy Beta $=0.356, \mathrm{p}=0.00<0.05$. Điều này có nghĩa là một nhân viên có sự hài lòng càng cao thì họ sẽ có những hành vi công dân tổ chức tốt trong công việc và ngược lại.

Nhận thức được sự hỗ trợ của tổ chức có tác động tích cực đến hành vi công dân tổ chức định hướng dịch vụ, từ kết quả hồi quy ta thấy trọng số hồi quy Beta $=0.228, \mathrm{p}=0.00<0.05$. Điều này có nghĩa là một nhân viên nhận thức được sự hỗ trợ của tổ chức là cao thì họ sẽ có những hành vi công dân tổ chức tốt và ngược lại. 


\section{Kết luận và hàm ý quản trị}

Dựa trên lý thuyết trao đổi xã hội kết hợp với nghiên cứu định tính, nghiên cứu xác định có 4 yếu tố tác động lên hành vi công dân tổ chức định hướng dịch vụ đối với nhân viên trong ngành hàng không đó là "Vốn tâm lý", "Sự hài lòng công việc", "Sự gắn kết công việc" và "Nhận thức được sự hỗ trợ của tổ chức".

\section{Hàm ý quản trị}

Từ kết quả nghiên cứu có thể dẫn đến một số hàm ý quản trị sau:

Các doanh nghiệp trong ngành hàng không nên có chính sách tiền lương hợp lý để thúc đẩy nhân viên của mình làm việc tốt hơn, gắn bó hơn với doanh nghiệp. Bên cạnh đó, doanh nghiệp cũng phải thường xuyên khảo sát sự hài lòng trong công việc của nhân viên để hiểu những mối quan tâm của họ, những áp lực trong công việc mà họ gặp phải đồng thời áp dụng hệ thống quản lý thích hợp (như chế độ lương, thưởng, phụ cấp, phúc lợi, cơ hội thăng tiến). Tạo dựng một số quỹ tích cực hỗ trợ nhân viên có hoàn cảnh khó khăn, nhân viên xuất sắc, ...

Để có thể nâng cao vốn tâm lý của nhân viên đầu tiên các doanh nghiệp cần chú trọng đến việc tuyển dụng các ứng viên đặc biệt là cần có bài kiểm tra về chỉ số $E Q$ (Emotional Quotient). Nếu nhân viên có chỉ số EQ cao thì họ có khả năng nhận biết, đánh giá và điều tiết cảm xúc của bản thân và mọi người rất tốt (Bradberry, Greaves, \& Lencioni, 2009). Bên cạnh đó trong quá trình làm việc, các doanh nghiệp nên đăng ký các khóa học nhằm tăng vốn tâm lý cho nhân viên của mình. Các khóa học này giúp nhân viên có kỹ năng giải quyết vấn đề một cách hiệu quả, giúp họ vượt qua thất bại và đưa ra các kế hoạch dự phòng cũng như tăng sự lạc quan cho nhân viên.

\section{Hạn chế của đề tài}

Tuy đã đạt được mục tiêu đề ra nhưng kết quả nghiên cứu vẫn còn một số hạn chế không tránh khỏi. Thứ nhất, sự giới hạn về nguồn tài liệu tham khảo đối với mảng đề tài "Hành vi công dân tổ chức định hướng dịch vụ" làm hạn chế việc lựa chọn các yếu tố ảnh hưởng đến khái niệm nghiên cứu cũng như xây dựng thang đo cho khái niệm. Thứ hai, phạm vi nghiên cứu chỉ giới hạn trong các doanh nghiệp của ngành hàng không, cần thiết phải có các nghiên cứu tương tự để kiểm chứng và so sánh đối chiếu kết quả nghiên cứu từ đó tìm ra đặc thù của từng ngành kinh tế khác nhau. Thứ ba, nghiên cứu chưa xem xét sự tác động qua lại giữa các biến độc lập với nhau vì theo như ma trận hệ số tương quan thì các biến độc lập đều có tương quan với nhau và với biến phụ thuộc.

\section{Tài liệu tham khảo}

Albrecht, S. (2010). Handbook of employee engagement. Cheltenham, England: Edward Elgar.

Ali, H. M. (2014). Perceived organizational support and organizational citizenship behavior: The case of Kuwait. International Journal of Business Administration, 5(3), 59-72.

Avey, J. B., Luthans, F., \& Jensen, S. M. (2009). Psychological capital: A positive resource for combating employee stress and turnover. Human Resource Management, 48(5), 677-693. 
Bakker, A. B. (2011). An evidence-based model of work engagement. Current Directions in Psychological Science, 20(4), 265-269.

Bateman, T. S., \& Organ, D. W. (1983). Job satisfaction and the good soldier: The relationship between affect and employee "citizenship". Academy of Management Journal, 26(4), 587-595.

Bettencourt, L. A., Gwinner, K. P., \& Meuter, M. L. (2001). A comparison of attitude, personality, and knowledge predictors of service-oriented organizational citizenship behavior and service quality. The Journal of Applied Psychology, 86(1), 29-41.

Bienstock, C. C., Demoranville, W. C., \& Smith, K. R. (2003). Organizational citizenship behavior and service quality. Journal of Services Marketing, 17(4), 357-378.

Blau, P. M. (1964). Exchange and power in social life. New York, NY: John Wiley.

Bowen, J., \& Ford, R. C. (2002). Managing service organizations: Does having a "thing" make a difference? Journal of Management, 28(3), 447-469.

Bradberry, T., Greaves, J., \& Lencioni, P. M. (2009). Emotional intelligence 2.0. San Diego, CA: TalentSmart.

Cranny, C. J., Smith, P. C., \& Stone, E. F. (1992). Job satisfaction: How people feel about their jobs and how it affects their performance. New York, NY: Lexington.

Eisenberger, R., Cummings, J., Armeli, S., \& Lynch, P. (1997). Perceived organizational support, discretionary treatment, and job satisfaction. Journal of Applied Psychology, 82(5), 812-820.

Eisenberger, R., Fasolo, P., \& Davis-LaMastro, V. (1990). Perceived organizational support and employee, diligence, commitment, and innovation. Journal of Applied Psychology, $75,51-59$

Eisenberger, R., Huntington, R., Hutchison, S., \& Sowa, D. (1986). Perceived organizational support. Journal of Applied Psychology, 71(3), 500-507.

Harris, C. (2012). Relationships betweens psychological capital, work engagement and organization citizenship behavior in South African automotive dealerships (Doctoral dissertation, Nelson Mandela Metropolitan University, South Africa). Retrieved September 12, 2018, from https://core.ac.uk/download/pdf/145048413.pdf

Ikonne, C. N. (2015). Job satisfaction and organizational citizenship behavior of library personnel in selected Nigerian universities. International Journal of Science and Research, 4(4), 2319-7064.

Kahn, W. A. (1990). Psychological conditions of personal engagement and disengagement at work. Academy of Management Journal, 33(4), 692-724.

Kamdar, D., \& Van Dyne, L. (2007). Theo joint effects of personality and workplace social exchange relationship in predicting task performance and citizenship performance. Journal of Applied Psychology, 92(5), 1286-1298.

Kang, H. J. (2014). A model of hospitality work engagement (Doctoral dissertation, University of Nevada, Las Vegas). Retrieved July 20, 2017, from https://digitalscholarship.unlv.edu/thesesdissertations/2102/ 
Khosravizadeh, O., Vatankhah, S., Alirezaei, S., Doosty, F., Esfahani, H. M., \& Rahimi, M. (2017). Organizational citizenship behavior and its relationship with psychological capital: A survey of hospital staff. Evidence Based Health Policy, Management \& Economics, 1(1), 24-31.

Ling, S. C. (2016). A study of the role of work engagement in promoting service-oriented organizational citizenship behavior in the Malaysian hotel sector. Global Business and Organizational Excellence, 35(4), 28-43.

Luthans, F., Avey, J. B., \& Patera, J. L. (2008). Experimental analysis of a web-based training intervention to develop positive psychological capital. Academy of Management Learning \& Education, 7(2), 209-221.

Luthans F., Avolio, B., Walumbwa, F., \& Li, W. (2005). The psychological capital of Chinese workers: Exploring the relationship with performance. Management and Organization Review, 1, 247-269.

Luthans, F., Avolio, B. J., Avey, J. B., \& Norman, S. M. (2007). Positive psychological capital: Measurement and relationship with performance and satisfaction. Personnel Psychology, 60, 541-572.

Luthans, F., \& Youssef, C. M. (2004). Human, social and now positive psychological capital management: Investing in people for competitive advantage. Organizational Dynamics, 33, 143-160.

Ma, E., \& Qu, H. (2011). Social exchanges as motivators of hotel employees' organizational citizenship behaviour: The proposition and application of a new three-dimensional framework. International Journal of Hospitality Management, 30, 680-688.

Manish, G. M. S., \& Prathap, K. R. (2017). Impact of psychological capital on organizational citizenship behavior. Journal of Management Development, 36(7), 973-983.

Mohammad, A. A., Jehad, M., \& Farzana, Q. H. (2011). Job satisfaction and organization citizenship behavior: An empirical study at higher learning institutions. Asian Academy of Management Journal, 16(2), 149-165.

Nguyen, T. D. (2013). Giáo trình nghiên cúu phương pháp khoa học trong kinh doanh [Scientific methodology study curriculum in business]. Ho Chi Minh City, Vietnam: Nhà xuất bản tài chính.

Organ, D. W. (1988). Organizational citizenship behavior: The good soldier syndrome. Lexington, MA: D. C. Heath.

Organ, D. W., \& Konovsky, M. (1989). Cognitive vs affective determinants of organizational citizenship behavior. Journal of Applied Psychology, 74(1), 157-164.

Podsakoff, P. M., \& MacKenzie, S. B. (1997). Impact of organizational citizenship behavior on organizational performance: A review and suggestions for future research. Human Performance, 10(2), 133-151. doi:10.1207/s15327043hup1002_5

Podsakoff, P. M., MacKenzie, S. B., Paine, J. B., \& Bacharch, D. G. (2009). Organizational citizenship behaviors: A critical review of the theoretical and empirical literature and suggestions for future research. Journal of Management, 26(3), 513-563. 
Prentice, C. \& King, B. E. M. (2011). The influence of emotional intelligence on the service performance of casino frontline employees. Tourism and Hospitality Research, 11(1), 4966. doi:10.1057/thr.2010.21

Rhoades, L., \& Eisenberger, R. (2002). Perceived organizational support: A review of the literature. Journal of Applied Psychology, 87(4), 698-714.

Saks, A. M. (2006). Antecedents and consequences of employee engagement. Journal of Managerial Psychology, 21(7), 600-619. doi:10.1108/02683940610690169

Schaufeli, W. B., Salanova, M., Gonzalez-Roma, V., \& Bakker, A. B. (2002). The measurement of burnout and engagement: A confirmatory factor analytic approach. Journal of Happiness Studies, 3(1), 71-92.

Schaufeli, W. B. (2012). Work engagement. What do we know and where do we go? Romanian Journal of Applied Psychology, 14, 3-10.

Schnake, M. E., \& Dumler, M. P. (2003). Levels of measurement and analysis issues in organizational citizenship behavior research. Journal of Occupational and Organizational Psychology, 76(3), 283-301.

Schriesheim, C., \& Tsui A. S. (1980). Development and validation of short satisfaction instrument for use in survey feedback interventions. Paper presented at the Western Academy of Management Meeting, Salt Lake City, Utah, USA.

Smith, C. A., Organ, D. W., \& Near, J. P. (1983). Organizational citizenship behavior: Its nature and antecedents. Journal of Applied Psychology, 68(4), 653-663.

Tsai, C.-T., \& Su, C.-S. (2011). Leadership, job satisfaction and service-oriented organizational citizenship behaviors in flight attendants. African Journal of Business Management, 5(5), 1915-1926.

Walumbwa, F. O., \& Schaubroeck, J. (2009). Leader personality traits and employee voice behavior: Mediating roles of ethical leadership and work group psychological safety. Journal of Applied Psychology, 94(5), 1275-1286. doi:10.1037/a0015848

Wu, C., \& Liu, N.-T. (2014). Perceived organizational support, organizational commitment and service-oriented organizational citizenship behaviors. International Journal of Business \& Information, 9(1), 61-88. 